01

\title{
Расчет высокочастотной электропроводности и постоянной Холла для тонкой металлической пленки
}

\author{
(C) И.А. Кузнецова, О.В. Савенко, А.А. Юшканов \\ Ярославский государственный университет им. П.Г. Демидова, \\ 150000 Ярославль, Россия \\ e-mail: kuz@uniyar.ac.ru
}

(Поступило в Редакцию 30 марта 2016 г.)

\begin{abstract}
Рассмотрена кинетическая задача о нахождении высокочастотной электропроводности и постоянной Холла для тонкой металлической пленки в поперечном стационарном магнитном поле и в продольном переменном электрическом поле. Предполагается диффузное отражение электронов от верхней и нижней границ пленки. На соотношение между толщиной пленки и длиной свободного пробега электронов ограничений не накладывается. Исследованы зависимости электропроводности и постоянной Холла от безразмерных параметров: частоты электрического поля, индукции магнитного поля и толщины пленки. Проведено сравнение полученных результатов с экспериментальными данными.
\end{abstract}

DOI: 10.21883/JTF.2017.12.45196.1831

\section{Введение}

Исследования свойств тонких проводящих пленок стимулированы бурным развитием нанотехнологий. Особый интерес уделяется проводящим пленкам толщиной 1-10 nm (сверхтонкие пленки) в силу специфичности их свойств. Такие пленки широко используются в микроэлектронике, в СВЧ электронике [1], нанофотонике, оптоэлектронике, в производстве солнечных элементов [2] и т.д. Поликристаллические пленки могут применяться при создании высокоемкостных конденсаторов с малой площадью обкладок [3]. Современные интегральные схемы содержат несколько миллиардов транзисторов, поэтому требования к качеству, параметрам и свойствам тонких пленок являются довольно высокими [4].

Нанотехнологии позволяют создавать материалы с характерным линейным размером порядка нанометров, тогда как длина свободного пробега носителей заряда в металлах варьируется в пределах $10-100 \mathrm{~nm}$, а в полупроводниках — в интервале $10-1000 \mathrm{~nm}[5,6]$, таким образом, практически реализуется ситуация, когда длина свободного пробега носителей заряда сравнима или больше толщины пленки. Из эксперимента следует, что для пленок серебра, золота, меди наблюдается резкая зависимость электропроводности и оптических характеристик, в частности коэффициента отражения, от толщины пленки при ее увеличении в диапазоне от 5 до $40 \mathrm{~nm}$ [7]. Поэтому теоретическое исследование электрических и оптических свойств тонких пленок остается актуальным. Если толщина пленки много больше длины волны де Бройля, которая в типичных металлах составляет величину порядка межатомного расстояния $(\sim 0.3 \mathrm{~nm})$, а в полупроводниках принимает значение $\sim 10 \mathrm{~nm}[5,6]$, то квантовыми эффектами в подобных задачах можно пренебречь.
Еще в XIX веке Фарадеем было установлено, что электропроводность тонких пленок может быть много меньше электропроводности массивных металлов. Данное явление было теоретически проанализировано Фуксом [8] и Зондгеймером $[9,10]$. В работах $[9,10]$ впервые рассмотрено влияние магнитного поля на статическую проводимость тонкой металлической пленки $[9,10]$ и тонкой цилиндрической проволоки [9].

В настоящей работе проводится исследование высокочастотной электропроводности тонкой металлической пленки, находящейся в поперечном магнитном поле и в продольном переменном электрическом поле. Предполагаем, что толщина пленки меньше глубины скин-слоя, что позволяет пренебречь скин-эффектом. Отметим, что скин-эффект учитывался в работе [11] для случая тонкой цилиндрической проволоки. Будем считать, что электрическое и магнитное поле однородны.

\section{Постановка задачи}

Рассмотрим тонкий металлический слой толщины а, находящийся в поперечном магнитном поле напряженности Н. К этому слою приложено однородное периодическое во времени электрическое поле напряженности $\mathbf{E}$, параллельное плоскости пленки и меняющееся по закону:

$$
\mathbf{E}=\mathbf{E}_{0} \exp (-i \omega t)
$$

где $\omega-$ частота переменного напряжения, приложенного к слою.

Если толщина пленки много больше длины свободного пробега электронов $(a \gg \lambda)$, то для решения данной задачи можно воспользоваться классической электроди- 
намикой, т. е. применим локальный закон Ома:

$$
\begin{gathered}
\mathbf{j}=\Sigma(\omega) \mathbf{E}, \\
\Sigma(\omega)=\sigma_{0} /(1-i \tau \omega),
\end{gathered}
$$

где $\Sigma(\omega)$ - проводимость Друде, $\sigma_{0}=n e^{2} \tau / m-$ статическая проводимость, $e$ и $m-$ соответственно элементарный заряд и масса электрона, $n-$ концентрация электронов, $\tau-$ время релаксации.

В случае, когда толщина пленки сравнима с длиной свободного пробега электронов или меньше ее $(a \leq \lambda)$, макроскопическая электродинамика становится неприменимой, и для решения задачи необходимо применять кинетический метод.

Электрическое поле (1) действует на электроны проводимости в пленке и вызывает отклонение их функции распределения от равновесной функции Ферми-Дирака:

$$
\begin{aligned}
f(\mathbf{v}, z, t) & =f_{0}(\varepsilon)+f_{1}(\mathbf{v}, z, t) \\
& =f_{0}(\varepsilon)+f_{1}(\mathbf{v}, z) \exp (-i \omega t),
\end{aligned}
$$

где $\varepsilon=m v^{2} / 2-$ кинетическая энергия электрона, $v-$ скорость электрона.

Равновесную функцию распределения электронов можно представить в виде ступенчатой аппроксимации

$$
f_{0}(\varepsilon)= \begin{cases}1, & 0<\varepsilon \leq \varepsilon_{F} \\ 0, & \varepsilon>\varepsilon_{F}\end{cases}
$$

где $\varepsilon_{F}=m v_{F}^{2} / 2$ - энергия Ферми, $v_{F}-$ скорость Ферми.

Функцию $f_{1}$ можно найти из уравнения Больцмана в приближении времени релаксации и в линейном по внешнему полю приближении [9]

$$
\begin{array}{r}
\frac{\partial f_{1}}{\partial z}+\frac{v}{v_{z}} f_{1}-\frac{e H}{m c v_{z}}\left(v_{y} \frac{\partial f_{1}}{\partial v_{x}}-v_{x} \frac{\partial-f_{1}}{\partial v_{y}}\right) \\
=\frac{e}{m v_{z}}\left(E_{x} \frac{\partial f_{0}}{\partial v_{x}}+E_{y} \frac{\partial f_{0}}{\partial v_{y}}\right),
\end{array}
$$

где $v=\tau^{-1}-i \omega-$ комплексная частота рассеяния.

Будем считать, что электроны отражаются от верхней и нижней границ пленки диффузно, т.е. неравновесная поправка $f_{1}$ к функции распределения отраженных от верхней и нижней границ пленки электронов равна нулю:

$$
\begin{cases}f_{1}^{+}(\mathbf{v}, 0)=0, & v_{z}>0 \\ f_{1}^{-}(\mathbf{v}, a)=0, & v_{z}<0 .\end{cases}
$$

Задача сводится к отысканию функции распределения электронов, у которых проекция скорости на ось $Z$ положительна $\left(f_{1}^{+}(\mathbf{v}, z)\right)$ и у которых проекция скорости на ось $Z$ отрицательна $\left(f_{1}^{-}(\mathbf{v}, z)\right)$.
Зная функцию распределения электронов, можно рассчитать ток, плотность которого определяется следующим образом:

$$
\mathbf{j}=-2 e\left(\frac{m}{h}\right)^{3} \int \mathbf{v} f_{1} d^{3} v
$$

Используя выражение (7), можно найти проводимость и постоянную Холла для пленки.

\section{Расчет проводимости и постоянной Холла для тонкой металлической пленки}

Уравнение Больцмана (5) можно решить способом, аналогичным [9]. Для этого представим функцию $f_{1}$ в следующем виде:

$$
f_{1}(\mathbf{v}, z)=\left[v_{x} c_{1}\left(v_{z}, z\right)+v_{y} c_{2}\left(v_{z}, z\right)\right] \frac{\partial f_{0}}{\partial v},
$$

где $c_{1}\left(v_{z}, z\right)$ и $c_{2}\left(v_{z}, z\right)$ - некоторые функции от $z$-й компоненты скорости и координаты $z$.

После подстановки (8) в уравнение (5) получим следующее выражение:

$$
\begin{aligned}
v_{x} & \frac{\partial c_{1}\left(v_{z}, z\right)}{\partial z}+v_{y} \frac{\partial c_{2}\left(v_{z}, z\right)}{\partial z} \\
& +\frac{v}{v_{z}}\left[v_{x} c_{1}\left(v_{z}, z\right)+v_{y} c_{2}\left(v_{z}, z\right)\right] \\
& -\frac{e H}{m c v_{z}}\left[v_{y} c_{1}\left(v_{z}, z\right)-v_{x} c_{2}\left(v_{z}, z\right)\right] \\
& =\frac{e}{m v v_{z}}\left(E_{x} v_{x}+E_{y} v_{y}\right) .
\end{aligned}
$$

Приравнивая коэффициенты при $v_{x}$ и $v_{y}$, получим систему дифференциальных уравнений для функций $c_{1}\left(v_{z}, z\right)$ и $c_{2}\left(v_{z}, z\right)$ :

$$
\left\{\begin{array}{l}
\frac{\partial c_{1}\left(v_{z}, z\right)}{\partial z}+\frac{e H}{m c v_{z}} c_{2}\left(v_{z}, z\right)+\frac{v}{v_{z}} c_{1}\left(v_{z}, z\right)=\frac{e E_{x}}{m v v_{z}}, \\
\frac{\partial c_{2}\left(v_{z}, z\right)}{\partial z}-\frac{e H}{m c v_{z}} c_{1}\left(v_{z}, z\right)+\frac{v}{v_{z}} c_{2}\left(v_{z}, z\right)=\frac{e E_{y}}{m v v_{z}} .
\end{array}\right.
$$

Плоскость пленки можно представить как комплексную плоскость, роль действительной оси которой играет ось $X$, а роль мнимой оси - ось $Y$. Введем новые переменные

$$
\begin{gathered}
g\left(v_{z}, z\right)=c_{1}\left(v_{z}, z\right)-i c_{2}\left(v_{z}, z\right), \\
F=E_{x}-i E_{y},
\end{gathered}
$$

где $F-$ комплексная напряженность электрического поля.

Система (10) сведется к одному уравнению

$$
\frac{\partial g\left(v_{z}, z\right)}{\partial z}+\frac{i e H}{m c v_{z}} g\left(v_{z}, z\right)+\frac{v}{v_{z}} g\left(v_{z}, z\right)=\frac{e F}{m v v_{z}}
$$


общее решение которого определяет функцию $g\left(v_{z}, z\right)$ :

$$
\begin{aligned}
g\left(v_{z}, z\right)= & \frac{e F}{m v v\left(1+\frac{i e H}{v m c}\right)} \\
& \times\left\{1+\psi\left(v_{z}\right) \exp \left[-\frac{z v}{v_{z}}\left(1+\frac{i e H}{v m c}\right)\right]\right\} .
\end{aligned}
$$

Здесь $\psi\left(v_{z}\right)$ - постоянная интегрирования, зависящая только от $v_{z}$. Функцию $\psi\left(v_{z}\right)$ можно найти из граничных условий (6):

$$
\begin{cases}\psi^{+}\left(v_{z}\right)=-1, & v_{z}>0, \\ \psi^{-}\left(v_{z}\right)=-\exp \left[\frac{a v}{v_{z}}\left(1+\frac{i e H}{v m c}\right)\right], & v_{z}<0 .\end{cases}
$$

Для нахождения плотности тока по формуле (7) удобно воспользоваться сферической системой координат в пространстве скоростей $(\mathbf{v}=(v, \theta, \gamma)$, где $v-$ модуль скорости, $\theta$ и $\gamma-$ соответственно полярный и азимутальный углы в пространстве скоростей).

Подставив функцию $f_{1}(8)$ в выражение (7), получим проекции плотности тока на ось $X$ и $Y$ :

$$
\begin{aligned}
& j_{x}=-2 \pi e\left(\frac{m}{h}\right)^{3} \int_{0}^{\infty} \int_{0}^{\pi} v^{4} \sin ^{3} \theta c_{1} \frac{\partial f_{0}}{\partial v} d v d \theta, \\
& j_{y}=-2 \pi e\left(\frac{m}{h}\right)^{3} \int_{0}^{\infty} \int_{0}^{\pi} v^{4} \sin ^{3} \theta c_{2} \frac{\partial f_{0}}{\partial v} d v d \theta .
\end{aligned}
$$

Введем новое обозначение $J=j_{x}-i j_{y}$, которое будем называть комплексным током, тогда (15) и (16) можно свести к одному выражению

$$
J=-2 \pi e\left(\frac{m}{h}\right)^{3} \int_{0}^{\infty} \int_{0}^{\pi} v^{4} \sin ^{3} \theta g\left(v_{z}, z\right) \frac{\partial f_{0}}{\partial v} d v d \theta .
$$

В экспериментальных условиях обычно находят усредненный по толщине пленки ток:

$$
\begin{aligned}
\bar{J}= & \frac{1}{a} \int_{0}^{a} J d z=-\frac{2 \pi e}{a}\left(\frac{m}{h}\right)^{3} \\
& \times \int_{0}^{a} \int_{0}^{\infty} \int_{0}^{\pi} v^{4} \sin ^{3} \theta g\left(v_{z}, z\right) \frac{\partial f_{0}}{\partial v} d v d \theta d z \\
= & -\frac{2 \pi e}{a}\left(\frac{m}{h}\right)^{3} \int_{0}^{a} \int_{0}^{\infty} v^{4} \frac{\partial f_{0}}{\partial v} \\
& \times\left\{\int_{0}^{\pi / 2} \sin ^{3} \theta g^{+}\left(v_{z}, z\right) d \theta \int_{\pi / 2}^{\pi} \sin ^{3} \theta g^{-}\left(v_{z}, z\right) d \theta\right\} d v d z .
\end{aligned}
$$

Подставляя функцию $g\left(v_{z}, z\right)$ в $(18)$ и проведя необходимые вычисления, получим выражение для комплексного тока, усредненного по толщине пленки:

$$
\begin{gathered}
\bar{J}=\frac{\sigma_{0} x_{0} F}{\varphi(s)}, \quad \sigma_{0}=\frac{n e^{2} \tau}{m} \\
\frac{1}{\varphi(s)}=\frac{1}{s}\left\{1-\frac{3}{8 s}+\frac{3}{2 s} \int_{1}^{\infty}\left(\frac{1}{t^{3}}-\frac{1}{t^{5}}\right) \exp (-s t) d t\right\} \\
t=\frac{1}{\cos \theta}, \quad s=z_{0}+i \beta \\
\beta=a \frac{e H}{m v_{F} c}, \quad z_{0}=\frac{a v}{v_{F}}=\frac{a}{v_{F} \tau}-i \frac{a \omega}{v_{F}}=x_{0}-i y_{0} .
\end{gathered}
$$

Используя выражение (19), найдем проводимость и постоянную Холла для пленки

$$
\begin{gathered}
\sigma=\frac{j_{x}}{E_{x}}=\frac{\sigma_{0} x_{0}}{E_{x}} \frac{E_{x} \operatorname{Re}(\varphi(s))-E_{y} \operatorname{Im}(\varphi(s))}{|\varphi(s)|^{2}} \\
A_{H}=\frac{E_{y}}{H j_{x}}=-\frac{E_{y} A_{H, 0}}{\beta} \frac{|\varphi(s)|^{2}}{E_{x} \operatorname{Re}(\varphi(s))-E_{y} \operatorname{Im}(\varphi(s))} .
\end{gathered}
$$

Здесь $A_{H, 0}=-1 /($ enc $)-$ постоянная Холла в классическом случае.

Предполагая, что ток вдоль оси $Y$ не течет $\left(j_{y}=\operatorname{Im}(\bar{J})=0\right)$, найдем связь между $x$ - и $y$-компонентами напряженности электрического поля:

$$
E_{y} \operatorname{Re}(\varphi(s))+E_{x} \operatorname{Im}(\varphi(s))=0 .
$$

Используя (23) и подставляя выражение (20) для функции $\varphi(s)$ в (21) и (22), получим окончательное выражение для проводимости и постоянной Холла тонкой металлической пленки

$$
\begin{gathered}
\sigma\left(x_{0}, y_{0}, \beta\right)=\sigma_{0} \Sigma\left(x_{0}, y_{0}, \beta\right), \\
A_{H}\left(x_{0}, y_{0}, \beta\right)=A_{H, 0} R_{H}\left(x_{0}, y_{0}, \beta\right),
\end{gathered}
$$

$$
\Sigma\left(x_{0}, y_{0}, \beta\right)=
$$

$$
=x_{0} \frac{\left(z_{0}-\frac{3}{8}(1-4 a)\right)^{2}+\left(\beta-\frac{3}{2} b\right)^{2}}{z_{0}\left(z_{0}^{2}+\beta^{2}\right)-3 b \beta z_{0}+\frac{3}{8}(1-4 a)\left(\beta^{2}-z_{0}^{2}\right)},
$$

$R_{H}\left(x_{0}, y_{0}, \beta\right)=$

$$
=\frac{1}{\beta_{0}} \frac{\frac{3}{2} b\left(z_{0}^{2}-\beta^{2}\right)-\frac{3}{4} \beta z_{0}(1-4 a)+\beta\left(z_{0}^{2}+\beta^{2}\right)}{\left(z_{0}-\frac{3}{8}(1-4 a)\right)^{2}+\left(\beta-\frac{3}{2} b\right)^{2}},
$$

$$
\begin{aligned}
& a=\int_{1}^{\infty}\left(\frac{1}{t^{3}}-\frac{1}{t^{5}} \exp \left(-z_{0} t\right) \cos (\beta t) d t,\right), \\
& b=\int_{1}^{\infty}\left(\frac{1}{t^{3}}-\frac{1}{t^{5}} \exp \left(-z_{0} t\right) \sin (\beta t) d t,\right) .
\end{aligned}
$$




\section{Предельные случаи}

1. Рассмотрим статический случай $\left(y_{0}=0\right)$. Выражения (20), (26) и (27) будут иметь тот же вид, если переменную $z_{0}$ заменить на $x_{0}$. В этом статическом пределе полученные выражения согласуются с результатами работ $[9,12]$.

2. Рассмотрим другой предельный случай, когда магнитное поле отсутствует $(\beta=0)$. Выражение (26) примет следующий вид:

$$
\Sigma\left(x_{0}, y_{0}\right)=\frac{x_{0}}{z_{0}}\left(1-\frac{3}{8 z_{0}}+\frac{3}{2 z_{0}} \int_{1}^{\infty}\left(\frac{1}{t^{3}}-\frac{1}{t^{5}}\right) \exp \left(-z_{0} t\right) d t\right) .
$$

Таким образом, безразмерная проводимость $\Sigma\left(x_{0}, y_{0}\right)(28)$ в отсутствие магнитного поля совпадает с результатом работы [13] при нулевых значениях коэффициентов зеркальностей верхней и нижней границ пленки.

3. В предельном случае $\left|z_{0}\right| \gg 1$ в выражениях (26) и (27) доминируют слагаемые с наибольшей степенью $z_{0}$, а экспоненциальными членами можно пренебречь. В результате получим классический результат $[14]$

$$
\begin{gathered}
\Sigma=\frac{x_{0}}{z_{0}}, \quad \sigma=\sigma_{0} \frac{x_{0}}{z_{0}}=\frac{\sigma_{0}}{1-i \omega \tau}, \\
R_{H}=1, \quad A_{H}=A_{H, 0}=-\frac{1}{e n c} .
\end{gathered}
$$

\section{Анализ полученных результатов}

На рис. 1, изображены частотные зависимости модуля (рис. $1, a)$ и аргумента (рис. $1, b)$ безразмерной проводимости тонкой металлической пленки (формула (26)) при значении безразмерной индукции магнитного поля $\beta=0$ (сплошные кривые) и $\beta=5$ (штриховые кривые) и при различных значениях безразмерной обратной длины свободного пробега электронов $x_{0}$. Из графиков видно, что зависимости модуля проводимости с увеличением безразмерной частоты электрического поля $y_{0}$ монотонно уменьшаются, а при больших значениях $y_{0}$ все кривые сливаются. Это объясняется тем, что электронный газ, не успевая откликнуться на высокочастотные колебания вектора напряженности электрического поля, ведет себя, в какой-то мере как совокупность связанных зарядов, которые не вносят вклад в проводимость. Аргумент проводимости с увеличением частоты электрического поля монотонно возрастает и стремится к $\pi / 2$, т. е. проводимость становится чисто мнимой величиной. Из графиков также видно, что в области относительно низких частот $\left(y_{0}<1\right.$ для модуля проводимости и $y_{0}<3$ для аргумента проводимости) при включении магнитного поля модуль и аргумент проводимости становятся больше, чем в отсутствие магнитного поля.


Рис. 1. Зависимости модуля $(a)$ и аргумента $(b)$ безразмерной проводимости $\Sigma$ от безразмерной частоты электрического поля $y_{0}$ при значении безразмерной индукции внешнего магнитного поля $\beta=0$ (сплошные кривые $1-4$ ) и $\beta=5$ (штриховые кривые $5-8): 1,5-x_{0}=0.1 ; 2,6-x_{0}=0.2 ; 3,7-x_{0}=0.3$; $4,8-x_{0}=0.5$.

На рис. 2 и 3 построены частотные зависимости модуля $(a)$ и аргумента $(b)$ безразмерной постоянной Холла (формула (27)) тонкой металлической пленки при значении безразмерной индукции внешнего магнитного поля $\beta=0.1$ (рис. 2) и $\beta=2$ (рис. 3). На этих рисунках наблюдаются осцилляции, которые усиливаются с уменьшением толщины пленки и увеличением длины свободного пробега электронов (уменьшением $x_{0}$ ). C увеличением частоты электрического поля эти осцилляции постепенно затухают. При совпадении безразмерной частоты электрического поля и безразмерной индукции внешнего магнитного поля значения модуля безразмерной постоянной Холла становятся близкими для всех значений $x_{0}$, и наблюдается резкое возрастание аргумента безразмерной постоянной Холла $\left(y_{0}=0.1\right.$ и $\beta=0.1$ на рис. $2 ; y_{0}=2$ и $\beta=2$ на рис. 3) при малых значениях $x_{0}$.

На рис. 4 построены зависимости модуля и аргумента безразмерной проводимости (формула (26)) от безразмерной индукции магнитного поля $\beta$ при различных значениях безразмерной обратной длины свободного пробега электронов $x_{0}$. Значения безразмерной частоты электрического поля $y_{0}=0$ и $y_{0}=0.5$ (рис. 4, $a$ ), а также $y_{0}=0.3$ и $y_{0}=0.5$ (рис. $4, b$ ). На этих рисунках наблюдаются осцилляции модуля и аргумента проводи- 

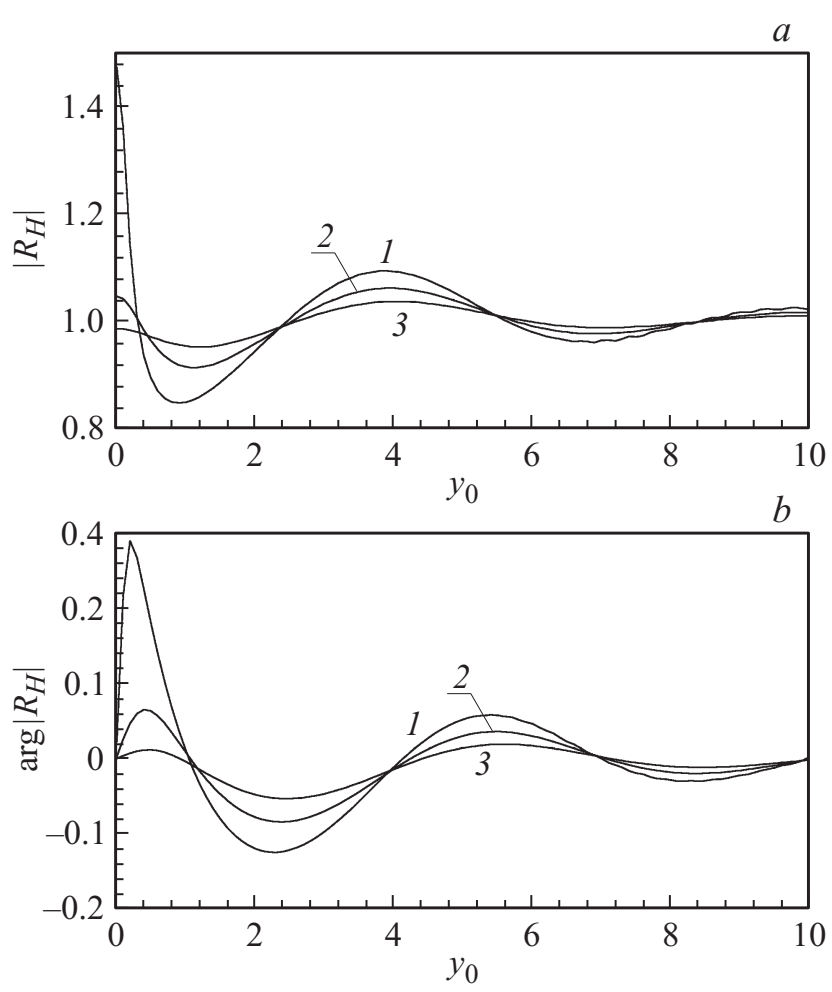

Рис. 2. Зависимости модуля $(a)$ и аргумента $(b)$ безразмерной постоянной Холла $R_{H}$ от безразмерной частоты электрического поля $y_{0}$ при значении безразмерной индукции внешнего магнитного поля $\beta=0.1: 1-x_{0}=0.1 ; 2-x_{0}=0.5 ; 3-x_{0}=1$.
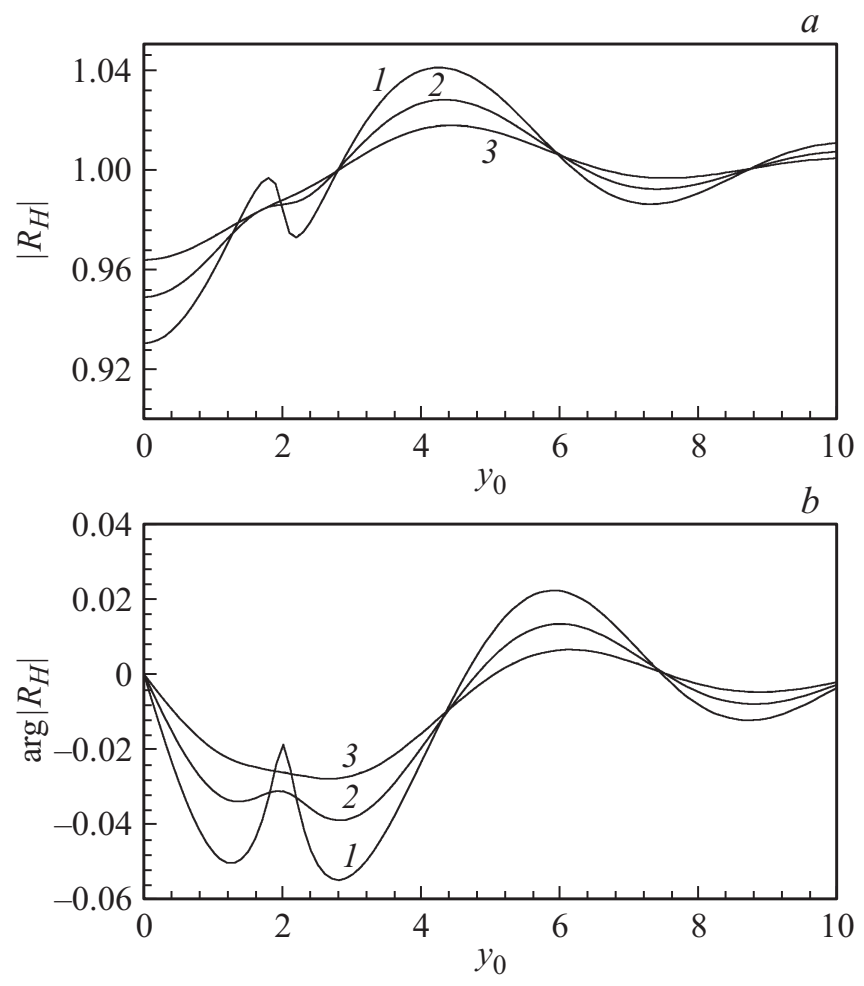

Рис. 3. Зависимости модуля $(a)$ и аргумента $(b)$ безразмерной постоянной Холла $R_{H}$ от безразмерной частоты электрического поля $y_{0}$ при значении безразмерной индукции внешнего магнитного поля $\beta=2: 1-x_{0}=0.1 ; 2-x_{0}=0.5 ; 3-x_{0}=1$.
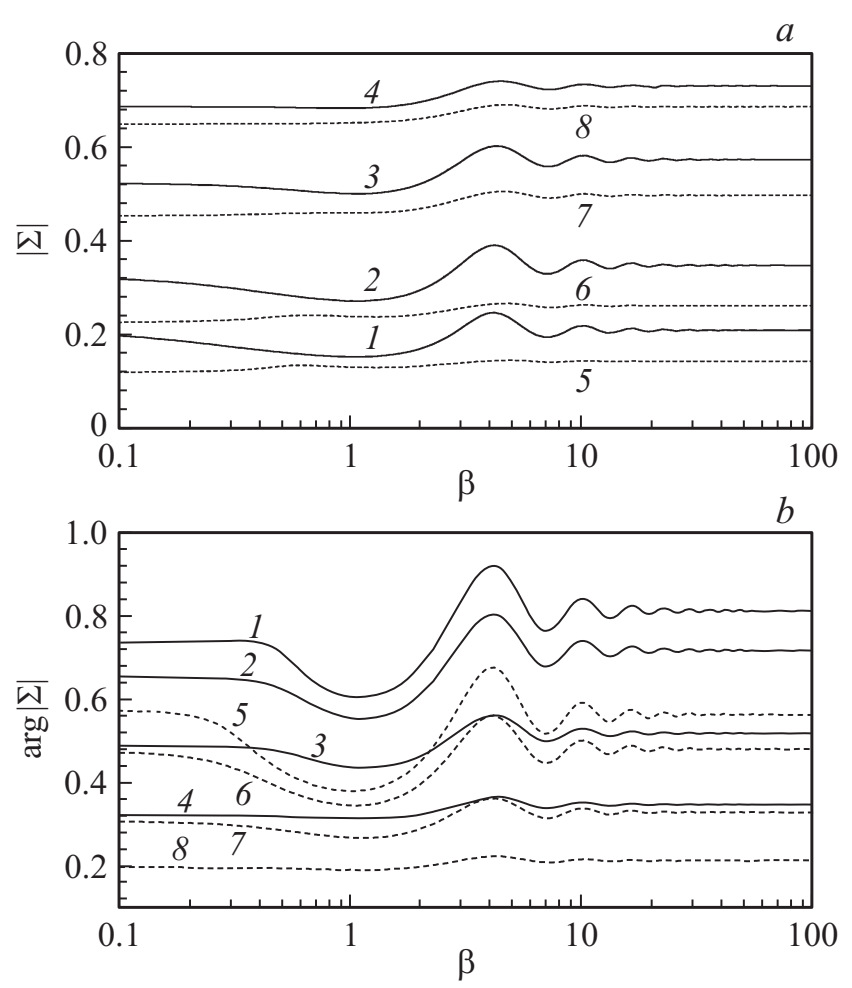

Рис. 4. Зависимости модуля $(a)$ и аргумента $(b)$ безразмерной проводимости $\Sigma$ от безразмерной индукции магнитного поля $\beta: a-$ сплошные кривые $-y_{0}=0$, штриховые кривые $-y_{0}=0.5 ; b-$ сплошные кривые $-y_{0}=0.3$, штриховые кривые $-y_{0}=0.5: 1,5-x_{0}=0.1 ; 2,6-x_{0}=0.2$; $3,7-x_{0}=0.5 ; 4,8-x_{0}=1$.

мости, которые затухают с увеличением безразмерной индукции магнитного поля $\beta$ и безразмерной обратной длины свободного пробега электронов $x_{0}$. В случае нестационарного электрического поля (штриховые кривые на рис. $4, a$ ) осцилляции модуля безразмерной проводимости исчезающе малы. Из рис. $4, b$ видно, что осцилляции аргумента проводимости начинаются, когда безразмерная индукция магнитного поля становится больше безразмерной частоты электрического поля.

На рис. 5 приведено сравнение экспериментальных и теоретических зависимостей тангенса угла Холла от индукции магнитного поля для четырех пленок золота различной толщины: 185, 150, 93 и $69 \mathrm{~nm}$ при температуре $50 \mathrm{~K}$. Экспериментальные данные взяты из работы [15], а теоретические кривые рассчитаны по формулам (24)-(27). Удельное сопротивление золота и длина свободного пробега электронов при температуре $50 \mathrm{~K}$ были рассчитаны по формуле Блоха-Грюнайзена [16]: $\rho_{50 \mathrm{~K}}=2.2 \cdot 10^{-7} \Omega \cdot \mathrm{cm}, \lambda=386 \mathrm{~nm}$ в предположении, что температура Дебая для золота равна $165 \mathrm{~K}$, а удельное сопротивление при температуре $273 \mathrm{~K}$ составляет $\rho_{273 \mathrm{~K}}=2.04 \cdot 10^{-6} \Omega \cdot \mathrm{cm}[16]$. Наблюдается различие в поведении теоретических и экспериментальных зависимостей, которое усиливается с увеличением индукции магнитного поля и уменьшением толщины пленки. Рас- 


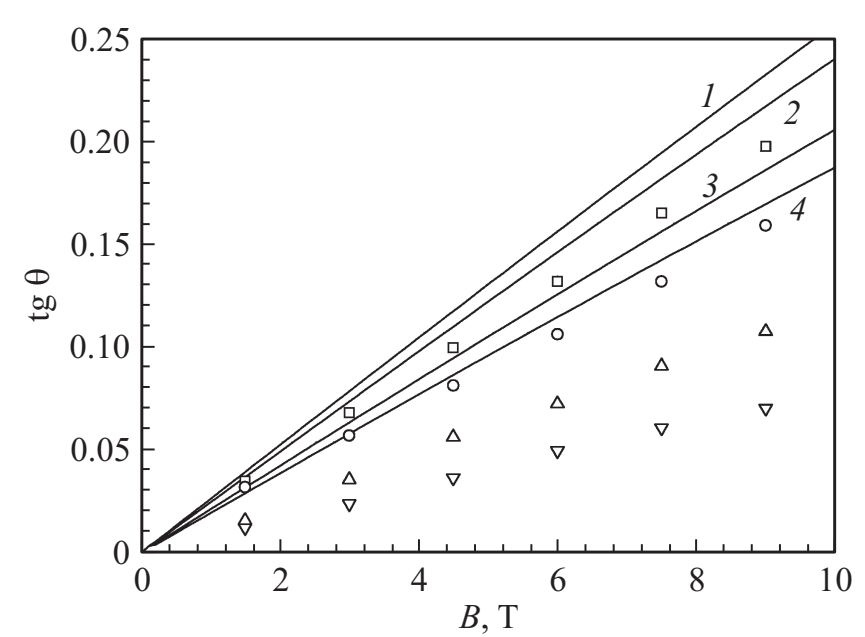

Рис. 5. Зависимости угла Холла от индукции магнитного поля для четырех пленок золота различной толщины. Сплошные кривые - теоретические зависимости, рассчитанные по формулам (24)-(27): 1, 2, 3, $4-185,150,93,69 \mathrm{~nm}$ coответственно. Точками обозначены экспериментальные данные работы [15]: $\square-185 \mathrm{~nm}$; ○ $-150 \mathrm{~nm} ; \triangle-93 \mathrm{~nm} ; \nabla-69 \mathrm{~nm}$.

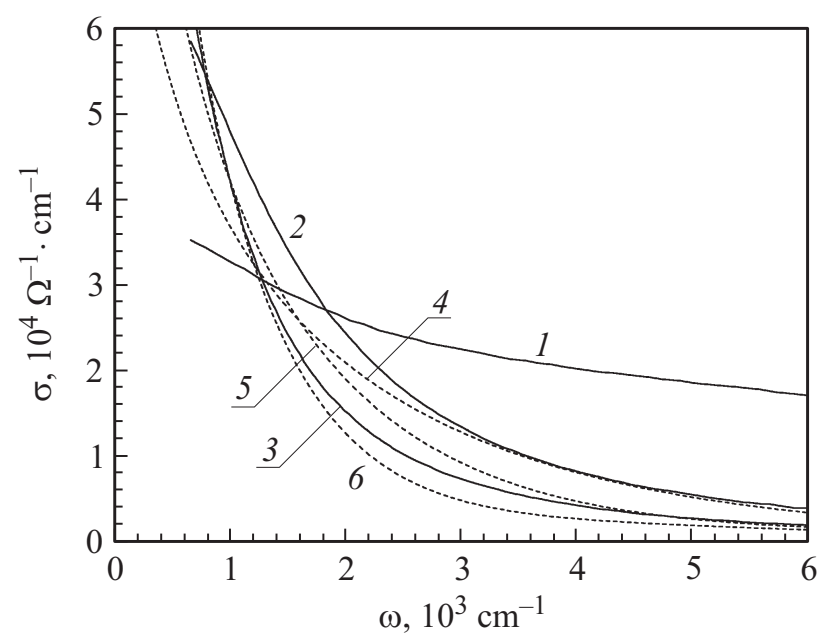

Рис. 6. Зависимости проводимости от частоты электрического поля для трех пленок золота различной толщины. Сплошные кривые $(1-3)$ - экспериментальные данные работы [17]; штриховые кривые $(4-6)$ - теоретические зависимости, рассчитанные по формулам (24) и (26): $1,4-3 \mathrm{~nm} ; 2,5-5 \mathrm{~nm}$; $3,6-9 \mathrm{~nm}$.

хождение теоретических и экспериментальных зависимостей можно объяснить тем, что исследуемые в работе [15] сплошные пленки имеют зернистую структуру, т. е. присутствует дополнительное рассеяние электронов на границах зерен, которое приводит к уменьшению проводимости с уменьшением толщины пленки.

На рис. 6 представлено сравнение теоретических и экспериментальных зависимостей проводимости от частоты падающей электромагнитной волны для трех пленок золота различной толщины $(9,5$ и $3 \mathrm{~nm})$ при комнатной температуре. Экспериментальные данные (сплошные кривые 1-3) были получены из работы [17]. Авторы данной работы измеряли коэффициент отражения пленки золота при различных частотах падающей электромагнитной волны, затем рассчитывали оптическую проводимость пленки для каждой частоты. Также построены теоретические зависимости (штриховые кривые 4-6), рассчитанные по формулам (24) и (26). Расчетные данные для золота [18]: удельная электропроводность при температуре $300 \mathrm{~K}$ без учета размерных эффектов $\sigma_{300 \mathrm{~K}}=4.35 \cdot 10^{5} \Omega^{-1} \cdot \mathrm{cm}^{-1}$, концентрация электронов $n=5.8 \cdot 10^{22} \mathrm{~cm}^{-3}$, длина свободного пробега электронов при температуре $300 \mathrm{~K} \lambda=37 \mathrm{~nm}$. Различие между теоретическими и экспериментальными кривыми увеличивается с уменьшением толщины пленки. Для пленки толщиной $3 \mathrm{~nm}$ различие в поведении теоретических и экспериментальных зависимостей становится существенным, что объясняется тем, что при толщинах пленки, меныших 4 nm [17], пленка становится островковой.

\section{Заключение}

Полученные результаты показали, что поверхностные эффекты оказывают существенное влияние на электрические свойства тонкой металлической пленки, толщина которой сопоставима или меньше длины свободного пробега электронов. Показано, что зависимости высокочастотной проводимости и постоянной Холла от таких параметров, как индукция магнитного поля, частота электрического поля и толщина пленки, ведут себя нетривиальным образом; в частности, обнаружены осцилляции зависимостей проводимости от индукции магнитного поля и постоянной Холла от частоты электрического поля.

\section{Список литературы}

[1] Касаткин Л.В., Чайка В.Е. Полупроводниковые устройства диапазона миллиметровых волн / Под ред. В.П. Тараненко, С.П. Ракитина. Севастополь: Вебер, 2006. 319 с.

[2] Андреев В.М., Давидюк Н.Ю., Малевски Д.А., Паньчак А.Н., Румяниев В.Д., Садчиков Н.А., Чекалин А.В., Lиquе A. // ЖТФ. 2014. Т. 84. Вып. 11. С. 72-79.

[3] Туриевич А.С., Ануфриев Л.П., Наливайко О.Ю., Лесникова В.П. // Доклады БГУИР. 2005. Т. 1. С. 87-92.

[4] Абелес $Ф$. Оптические свойства металлических пленок. Физика тонких пленок / Под ред. М.К. Франкомба, Р.У. Гофмана. М.: Мир, 1973. Т. 2. 392 с.

[5] Ансельм А.И. Введение в теорию полупроводников М: Наука, 1978. 616 с.

[6] Лифиии И.М., Азбель М.Я., Каганов М.И. Электронная теория металлов. М.: Наука, 1971. 415 с.

[7] Антонеи И.В., Котов Л.Н., Некипелов С.В., Карпушов Е.Н. // ЖТФ. 2004. Т. 74. Вып. 11. С. 102-106.

[8] Fuchs K. // Proc. Camb. Phil. Soc. 1938. Vol. 34. P. 100-108.

[9] Sondheimer E.H. // Advances in Physics. 2001. Vol. 50. N 6. P. $499-537$.

[10] Sondheimer E.H. // Phys. Rev. 1950. Vol. 80. P. 401-406. 
[11] Завитаев Э.В., Русаков О.В., Юшканов А.А. // ФТТ. 2012. Т. 54. Вып. 6. С. 1041-1047.

[12] Grishin A.M., Lutsishin P.P., Ostroukhov Yu.S., Panchenko O.A. // JETP. 1979. Vol. 76. N 4. P. 673682.

[13] Уткин А.И., Юиканов А.А. // Опт. и спектр. 2014. Т. 117. № 4. C. $650-654$.

[14] Ландау Л.Д., Лифиии Е.М. Теоретическая физика. М: Физматлит, 2005. Т. 8. 656 с.

[15] Munoz R.C., García J.P., Henríquez R., Moncada A.M., Espinosa A., Robles M., Kremer G., Moraga L., Cancino S., Morales J.R., Ramírez A., Oyarzún S., Suárez M.A., Chen D., Zumelzu E., Lizama C. // Phys. Rev. Lett. 2006. Vol. 96. P. 206803.

[16] Малков М.П., Данилов И.Б., Зельдович А.Г., Фрадков А.Б. Справочник по физико-техническим основам криогеники. М.: Энергоатомиздат, 1985. 432 с.

[17] Brandt T., Növel M., Gompf B., Dressel M. // Phys. Rev. B. 2008. Vol. 78. P. 205409.

[18] Кикоин И.К. Таблицы физических величин. М.: Атомиздат, 1976. $1008 \mathrm{c}$ 